\section{Introducing physical care standards into the MRCPsych curriculum}

With the recent change in the format of the MRCPsych examinations, it seems an opportune time to consider a more radical change to the curriculum: specifically, the inclusion of physical healthcare syllabic content as part of the core medical knowledge items.

Why is it that when the core curriculum for all acute medicine/medicine trainees (Federation of the Royal Colleges of Physicians, 2007) contain mandatory components dealing with psychiatric illness, do we feel that we are exempt from including physical health from our membership exams?

I have often heard colleagues bemoan the lack of awareness of mental health issues among non-psychiatric medical colleagues, but I feel it is incumbent on all of us to get our own collegiate house in order first.

Although we all have experience of dealing with physical health problems as part of our undergraduate training, and a minimum of 1 year's postgraduate experience, the skills and knowledge gained decay rapidly when used infrequently.

Perhaps the introduction of core topics into the MRCPsych curriculum such as the management of acute medical problems and the longer-term physical health problems associated with psychotropic use would go some way to remedy this.

\section{FEDERATION OF THE ROYAL COLLEGES OF} PHYSICIANS (2007) The Physician of Tomorrow. Curriculum for Core Medical Training and Acute Care Common Stem (Medicine) (Acute Medicine Level One). Federation of Royal Colleges of Physicians UK.

Ayesha Rahim ST4 Trainee in Psychiatry, Greater Manchester West Foundation Trust, Meadowbrook Unit, Stott Lane, Salford, Manchester M6 8HD, email: ayesha.rahim@doctors.org.uk

doi: 10.1192/pb.32.10.395

\section{Psychiatrists and role of religion in mental health}

Koenig (Psychiatric Bulletin, June 2008, 32, 201-203) highlights many aspects of understanding patients' religious beliefs with which I totally agree. However, it seems he has underestimated the influence of experience in psychiatric management. The statistical findings presented were the result of surveys which can never replace high-quality evidence-based research or well-designed qualitative studies. Psychiatrists in the UK have been under enormous strain to maintain a fine balance between legal and clinical responsibilities. The results of
Koenig's suggestions are not measurable and could raise a variety of medico-legal issues; for example, how to decide which patients should be involved with their psychiatrists for joint prayer? I believe this could be dangerous ground upon which to tread. Religion is a personal activity and psychiatrists could explore the interface between religious beliefs and psychopathology. But, they should definitely not cross sensitive boundaries.

Nazar Mansour Specialist Registrar (LAT) in Old Age Psychiatry, 15 Watery Lane, Northolt UBS 5QL, email: mansour_nazar@yahoo.co.uk

\section{doi: 10.1192/pb.32.10.395a}

Koenig's message (Psychiatric Bulletin, June 2008, 32, 201-203) is very clear for psychiatrists that they should respect patients' religious beliefs and a sensible way to address this is through time investment in taking a spiritual history, respecting and supporting patients' beliefs. Challenging beliefs and referrals to clergy should be welcomed but praying with patients is highly controversial and should be treated with caution.

There is a fine line between religiosity and religious conviction becoming a part of a complex delusional system. In clinical experience some patients are not religious prior to the onset of their mental illness. For such patients, becoming religious may be indicative of a relapse of their mental illness.

Religion and psychiatry are usually considered as two totally different ways of healing. A number of UK, US and Canadian studies confirm that psychiatrists are less likely to be religious in general, and are more likely to consider themselves spiritual rather than religious. Religious physicians are less willing than non-religious physicians to refer patients to psychiatrists (Curlin et al, 2007). The Australian experience is not different either (D'Souza et al, 2006).

CURLIN, F. A., ODELL, S.V., LAWRENCE, R. E., et a (2007) The relationship between psychiatry and religion among US physicians. Psychiatric Services, 58, 1193-1198.

D'SOUZA, R. \& GEORGE, K. (2006) Spirituality, religion and psychiatry: its application to clinical practice. Australasian Psychiatry, 14, 408-412.

*Imran Mushtaq Associate Specialist Child and Adolescent Psychiatrist, Sp-CAHMS, Eaglestone Centre, Standing Way, Milton Keynes MK6 5AZ, email: imranmushtaq@doctors.org.uk, Mohammad Adnan Hafeez Forensic Psychiatrist Edenfield Centre, Greater Manchester West Mental Health NHS Trust

doi: 10.1192/pb.32.10.395b
My one-time colleague and spiritual brother Rob Poole et al have expressed opinions which are to be respected (Psychiatric Bulletin, September 2008, 32, 356 -357). However, they may be in error when claiming that Koenig's editorial (Psychiatric Bulletin, June 2008, 32, 201203) is attempting 'to shift the boundaries of psychiatry'. There are no such boundaries, only pseudo-boundaries. Although human experience can be thought of in terms of physical, biological, psychological, social and spiritual dimensions (Culliford, 2002), there are no limits to or rigid cut-offs between them. They are indivisibly interconnected. Psychiatrists acknowledge continuous, fluid and potent interactions between the realms of biological brain and psychological mind, also between minds and society. Why not therefore recognise equally powerful, frequently healthy and therefore relevant movements of energy between minds and souls or whatever we experience as spiritual?

The eminent psychiatrist George Vaillant has recently, for example, written about the close relationship between spiritual experience and positive emotions like joy and hope (Vaillant, 2008). I have marshalled elsewhere (Culliford, 2007a) some arguments in favour of paying attention to the spiritual lives of psychiatric patients. To avoid doing so risks two important things: first, missing opportunities to improve rapport ('getting alongside patients' to use Poole et al's terminology) and patient adherence; and second, clinicians missing similar opportunities for additional personal growth through the reciprocal effects of compassionate intervention. Healthcare is a two-way process, and I have described in my book Love, Healing and Happiness (Culliford, 2007b) how this kind of growth comes about. Poole et al need not be too alarmed because none of this necessarily has anything to do with religion. In developing a non-denominational language of spirituality (using terms like 'spiritual awareness', 'spiritual practices', spiritual values' and 'spiritual skills') members of the Spirituality and Psychiatry Special Interest Group have taken pains to avoid some of the risks they outline. I look forward to continuing the 'serious debate' for which they say 'there is an urgent need'. To repeat, however, where boundaries do not exist, they cannot be blurred.

\section{Declaration of interest}

Larry Culliford is on the executive committee of the College's Spirituality and Psychiatry Special Interest Group. He has been paid to lecture on spirituality and mental healthcare, spirituality and healthcare, also spirituality and education. Until 2008, he taught a student-selected 